\title{
Underlying Venous Pathology Causing Perimesencephalic Subarachnoid Hemorrhage
}

\author{
Jaejoon Lee, Eun-Mi Koh, Chin-Sang Chung, Seung-Chul Hong, Yong-Bum Kim, \\ Pil-Wook Chung, Bum-Chun Suh, Heui-Soo Moon
}

\begin{abstract}
Background: Perimesencephalic subarachnoid hemorrhage (PSH) is a relatively benign clinical entity with a low risk of recurrent bleeding. The precise etiology of PSH has not yet been determined. We report here three cases of PSH with clinical and radiological features that support a venous system as a cause. Case Presentation: The first patient, a 72-year-old woman, had PSH and venous hemorrhagic infarct in the left thalamus on non-contrast CT. Subsequent cerebral angiography revealed widespread thrombosis in the cerebral venous system, a potential cause for reflux overflow hemorrhage. The second patient, a 55-year-old man with an established diagnosis of neuro-Behçet's disease, a well-known cause for cerebral venulitis, presented with PSH one year later. The third patient, a 39-year-old female, with incomplete Behçet's disease was admitted with PSH. Discussion: Current concepts on the anatomic origin and the possible pathophysiologic mechanism leading to PSH are discussed. The underlying pathological conditions in the venous system in our cases provide theoretical clues to the anatomic origin of PSH in general.
\end{abstract}

RÉSUMÉ: Pathologie veineuse sous-jacente causant une hémorragie sous-arachnoïdienne périmésencéphalique. Contexte : L'hémorragie sousarachnoïdienne périmésencéphalique (HSP) est une entité clinique relativement bénigne comportant un risque faible de saignement récurrent. L'étiologie précise de l'HSP n'a pas encore été déterminée. Nous rapportons trois observations d'HSP ainsi que les manifestations cliniques et radiologiques qui sont en faveur d'un lien de causalité avec le système veineux. Observations : La première patiente, une femme âgée de 72 ans qui a subi une HSP, présentait un infarctus hémorragique veineux dans le thalamus gauche à la tomodensitométrie sans substance de contraste. Des angiographies subséquentes ont montré une thrombose étendue du système veineux cérébral, une cause potentielle de l'hémorragie par regorgement. Le second patient, un homme de 55 ans porteur d'un diagnostic de neuro-Behçet, une cause bien connue de venulite cérébrale, a consulté pour une HSP un an plus tard. La troisième patiente, une femme âgée de 39 ans atteinte d'une maladie de Behçet incomplète, a été hospitalisée pour une HSP. Discussion : Nous discutons des concepts actuels sur l'origine anatomique et le mécanisme physiopathologique possible de l'HSP. Les conditions pathologiques sous-jacentes dans le système veineux chez nos patients fournissent des indices théoriques sur l'origine anatomique de l'HSP en général.

Can. J. Neurol. Sci. 2009; 36: 638-642

Perimesencephalic subarachnoid hemorrhage (PSH) has become well recognized as a distinct type of subarachnoid hemorrhage ( $\mathrm{SAH})$. This clinical entity of unknown etiology is defined as blood in the cisterns around the midbrain. Perimesencephalic subarachnoid hemorrhage constitutes approximately $10 \%$ of all patients with SAH and two thirds of those with a normal angiogram ${ }^{1-3}$. Patients with PSH have an excellent outcome without increased morbidity or mortality from recurrent bleeding and ischemia ${ }^{3-5}$. Both venous and arterial sources of bleeding have been suggested ${ }^{3,5,6}$ to explain this distinct subset of SAH. Definitive evidence supporting either of these speculations, however, still remains elusive. We report here three cases of PSH that most likely have arisen from an underlying abnormality in the venous system. The presence of underlying venous pathology predisposed the draining venules to rupture, ultimately leading to PSH. The authors speculate that the underlying venous pathology in our cases could provide theoretical clues to the anatomic origin of PSH in general.

\section{CASE Report}

\section{Patient 1}

A 72-year-old woman with a history of hypertension and diabetes mellitus was admitted to our institution for the evaluation of subacute onset of headache associated with vomiting. The patient also presented with progressive deterioration of mental status and clonic seizures of the left arm.

\footnotetext{
From the Department of Medicine (JL, EMK), Department of Neurology (CSC), Department of Neurosurgery (SCH), Samsung Medical Centre; Department of Neurology (YBK, PWC, BCS, HSM), Kangbuk Samsung Hospital, Sungkyunkwan University School of Medicine, Seoul, Republic of Korea.

Received February 17, 2009. Final Revisions Submitted April 29, 2009. Correspondence to: Heui-Soo Moon, Department of Neurology, Kangbuk Samsung Hospital, Sungkyunkwan University School of Medicine, Seoul, Republic of Korea.
} 


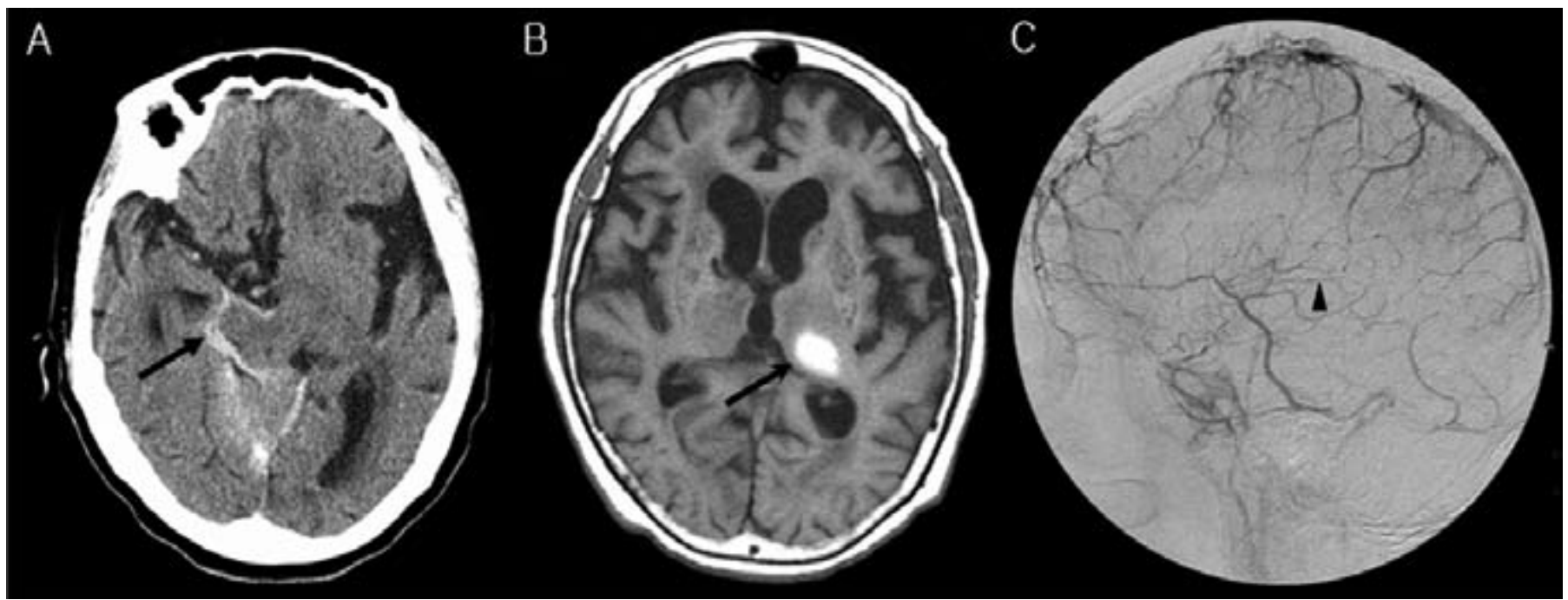

Figure 1: (A); The non-contrast brain CT showed a small amount of perimesencephalic subarachnoid hemorrhage. (B); T1-weighted MRI shows venous hemorrhagic infarct in the left thalamus. (C); Cerebral angiography reveals widespread thrombosis in the straight sinus, right transverse sinus, sigmoid sinus, and vein of Galen.

The blood pressure on admission was 160/86 mmHg. Neurologic examination revealed drowsiness, right hemiparesis and neck stiffness. Laboratory examinations, including complete blood count $(\mathrm{CBC})$, erythrocyte sedimentation rate (ESR), serum electrolytes, liver function tests, and coagulation studies were all within normal limits. Laboratory studies for the presence of an hypercoagulable state, including protein $\mathrm{C}$ and $\mathrm{S}$, antithrombin III, and antiphospholipid antibodies were all negative. Noncontrast computed tomography (CT) of the head showed a small amount of SAH in the perimesencephalic space (Figure 1A). Magnetic resonance imaging (MRI) performed five days after admission demonstrated hemorrhagic infarction with mass effect in the right thalamus (Figure 1B). Cerebral angiography revealed no aneurysm, vasospasm, stenoocclusive lesions or abnormal collateral vessels, but widespread thrombosis was present in the cerebral venous system, including the straight sinus, right transverse sinus, sigmoid sinus and vein of Galen (Figure 1C). A diagnosis of nonaneurysmal PSH was established. Electroencephalography revealed bilateral periodic lateralized epileptiform discharges. Mannitol was initiated for the control of suspected increased intracranial pressure. Prophylactic nimodipine was administered for prevention of vasospasm. Phenytoin was also given for seizure activity. Anticoagulation with intravenous heparin was held due to SAH and hemorrhagic transformation within the right thalamus. After the resolution of hemorrhage, an antiplatelet agent was administered. Although recurrent seizures subsided, the patient remained severely disabled and was discharged home after one month.

\section{Patient 2}

Approximately one-year prior to current hospitalization, a 55year-old man presented to our facility with transient global amnesia, visual disturbance and fever. On admission, body temperature was $38.1^{\circ} \mathrm{C}$ and other vital signs were normal. On physical examination, an aphthous ulcer was present on the buccal mucosa. Erythema nodosum on both shins were also found. Ophthalmologic examination was performed for visual disturbance which showed bilateral uveitis. Gait disturbance and ataxia on the left side were newly noticed shortly after the admission. Brain MRI showed asymmetrical areas of high signal intensity on T2-weighted sequences without mass effect, involving bilateral cerebellar dentate nuclei, pontine tegmentum, red nuclei of the midbrain, basal ganglia, and left median thalamus (Figure 2A). Lumbar puncture yielded clear cerebrospinal fluid under normal pressure with $81 \mathrm{mg} / \mathrm{dl}$ of glucose, $39 \mathrm{mg} / \mathrm{dl}$ of protein, and 19 leukocytes $/ \mathrm{mm}^{3}(47 \%$ lymphocytes, $14 \%$ polymorphonuclear leukocytes [PMNs]). Gram stains, acid fast bacilli (AFB) stain and virologic studies were negative. The diagnosis of neuro-Behçet's disease was established based on the recurrent oral ulcer, uveitis, erythematous skin lesion and compatible MRI findings. Treatment with methylprednisolone, one gram daily for five consecutive days was administered with prompt clinical improvement. Follow-up MRI, obtained seven days after the initiation of pulse prednisolone therapy, showed high intensity lesions involving the cerebellar dentate nucleus and the basal ganglia without changes from the previous study. The lesions in the pontine tegmentum and the midbrain which were present on previous MRI had disappeared. Prednisone $60 \mathrm{mg}$ /day was continued and slowly tapered over the next four months.

The patient was readmitted to our institution a year later due to severe headache and brief loss of consciousness after taking a steam bath. On admission, neurological examination was normal except nuchal rigidity. Brain CT revealed subarachnoid hemorrhage in the perimesencephalic region with small amounts of hemorrhage in the third ventricle (Figure 2B). The patient 


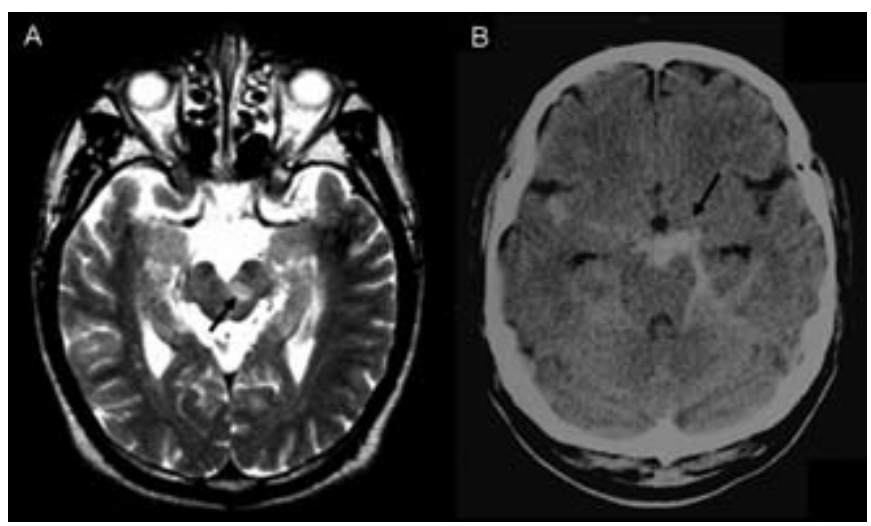

Figure 2: (A); Brain MRI shows high signal intensity involving the red nucleus of midbrain. (B); Brain CT reveals subarachnoid hemorrhage in the prepontine and perimesencephalic region.

underwent cerebral angiography, which demonstrated no vascular aneurysm or vasospasm. The venous phase digital subtraction angiography did not reveal any evidence of a vasculitis or venous abnormalities. Erythrocyte sedimentation rate and C-reactive protein were elevated at $45 \mathrm{~mm} / \mathrm{hr}$ (normal range $0-22 \mathrm{~mm} / \mathrm{hr}$ ) and $3.26 \mathrm{mg} / \mathrm{dl}$ (normal $0-0.3 \mathrm{mg} / \mathrm{dl}$ ), respectively. Cerebrospinal fluid (CSF) study showed pleocytosis $\left(3000 \mathrm{RBC} / \mathrm{mm}^{3}, 110 \mathrm{WBC} / \mathrm{mm}^{3}\right)$ with mildly increased protein and normal glucose. An insidious onset of a moderate headache associated with vomiting and fever appeared seven days after hospitalization. High dose of prednisolone was administered with resolution of clinical symptoms within a few days. The patient was discharged in an excellent condition after three weeks of hospital stay.

\section{Patient 3}

A 39-year-old female presented with an acute onset of headache, nausea and emesis of five days duration. Cognitive decline with increased drowsiness was reported by the family members. Past medical history included several episodes of painful oral ulcers in the past year and an acute onset of bilateral ocular redness which was self-limited. A history of genital ulcers history was absent. The patient was taking no medications. On physical examination, the patient appeared lethargic. A papulopustular skin lesion was noted at the area of previous venupuncture. No focal neurologic defect was noted and the brain stem reflexes including corneal, vestibulo-ocular, gag, and papillary light reflex were intact. Nuchal rigidity and positive Babinski's sign were present. Laboratory analysis revealed normal CBC and electrolytes. Hypercoagulability work-up was normal. Erythrocyte sedimentation rate and C-reactive protein were elevated at $33 \mathrm{~mm} / \mathrm{hr}$ and $2.34 \mathrm{mg} / \mathrm{dl}$, respectively. Cerebrospinal fluid (CSF) study revealed an opening pressure of $39 \mathrm{cmH}_{2} \mathrm{O}, 2 \mathrm{WBC} / \mathrm{mm}^{3}, 378 \mathrm{RBC} / \mathrm{mm}^{3}$, protein $134 \mathrm{mg} / \mathrm{dL}$, and glucose $61 \mathrm{mg} / \mathrm{dL}$ with serum glucose level of $119 \mathrm{mg} / \mathrm{dL}$. Brain $\mathrm{CT}$ revealed a small amount of $\mathrm{SAH}$ in the right perimesencephalic space and the third ventricle, along with low attenuation signals seen in the internal capsule, right thalamus, right external capsule and cerebellum (Figure 3A). On FLAIR MRI, increased signal involving bilateral basal ganglia, internal capsule, bilateral thalami, right medial temporal lobe, midbrain, and the right cerebellum were noted. Thrombosis was present in the straight sinus and the vein of Galen. Cerebral angiography showed no aneurysm or venospasm, but demonstrated occlusion of the inferior sagittal sinus, the straight sinus and vein of Galen (Figure 3B). Anticoagulation therapy was initiated along with pulse steroid therapy for five days. The follow-up MR venogram showed partial resolution of venous thrombi in the vein of Galen. The patient's clinical status improved gradually with mild residual dysarthria and memory deficit.

\section{Discussion}

The cause of perimesencephalic nonaneurysmal subarachnoid hemorrhage remains unknown and there exists much debate regarding the anatomical source of hemorrhage in PSH. The hemorrhage has been assumed to be either due to rupture of fine arterial vessels or a venous tear ${ }^{3,5,7}$. van Gijn et al postulated a non-arterial source of the hemorrhage, either venous or capillary $\mathrm{leak}^{5}$, and assumed a tear in the basal vein of Rosenthal (BVR) or one of its tributaries as the most likely source. The main arguments for a venous leak have been the limited extension of the hemorrhage, the invariably mild clinical features at onset, and no clinical deterioration from rebleeding or delayed cerebral ischemia $^{3,5-8}$.

Aberrant venous flow appears to play a role in the development of PSH. van der Schaaf et al reported that, in patients with PSH, venous drainage is often directly into dural sinuses instead of via the vein of Galen and this primitive variant of venous drainage is much more frequent in patients with PSH than those with an aneurysmal SAH (A-SAH $)^{9}$. Furthermore, in patients with unilateral primitive variant, the blood was always found on the side of the primitive drainage, suggesting an association between the primitive venous variant and bleeding 9 . The reason for this observation remains unclear. It has been suggested that the direct communication of perimesencephalic

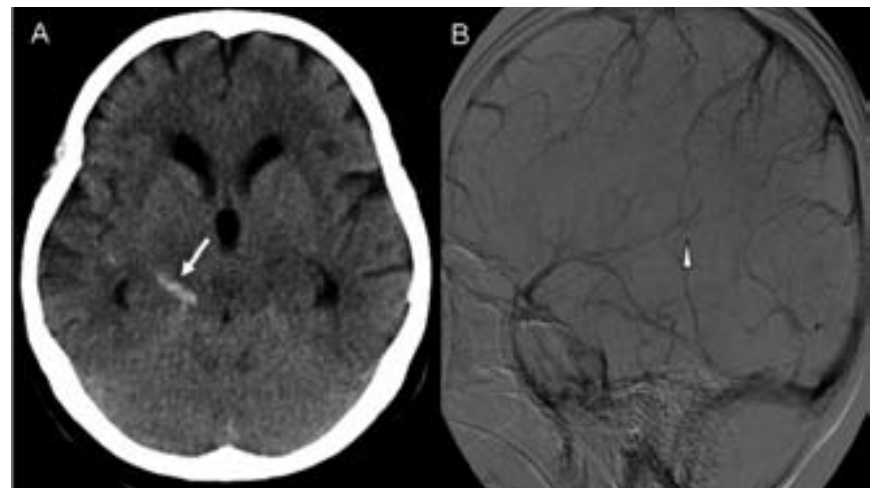

Figure 3: (A); Brain CT reveals a small amount of $S A H$ in perimesencephalic space. (B); Cerebral angiography shows occlusion in the inferior sagittal sinus, the straight sinus and the vein of Galen. 
veins with the dural sinuses may predispose to sudden increases in venous pressure with engorgement and rupture of the veins as a result ${ }^{10}$. A similar observation was reported by Yamakama et al in which the venograms of patients with PSH were compared with those of patients with A-SAH, particularly focusing on the $\mathrm{BVR}^{11}$. The location and drainage pathway of the BVR was found to be a significantly more primitive configuration in patients with PSH than in those with A-SAH. He also suggested that failure of longitudinal anastomoses between the primary primitive veins as well as excessive strenuous exertion including components of the Valsalva maneuver plays an important predisposing role in the etiology of PSH. Altogether, these results indicate a venous origin of PSH.

On the other hand, in a controlled study by Daenekindt et al involving 59 patients with $\mathrm{PSH}$ and 59 patients with A-SAH, the presence of a primitive variant of the BVR was not significantly different between these group ${ }^{12}$. Furthermore, it is speculated that a lesion of the basilar artery is responsible for the $\mathrm{SAH}^{13}$, and the rupture of a perforating artery has been also suggested to explain some instances of $\mathrm{PSH}^{14}$. Despite these numerous hypothetical postulations, the exact anatomical origin of $\mathrm{PSH}$ still remains to be elucidated.

Our first patient had PSH with direct evidence of wide spread venous thrombosis on cerebral angiography. Reports of SAH in association with cerebral venous thrombosis is very rare ${ }^{15}$. A feasible explanation for PSH in the first patient is that the thrombosis of the deep cerebral venous system including the great vein of Galen and straight sinus may have exerted retrograde hydrostatic pressure into the veins which bridge the perimesencephalic subarachnoid space. The veins in this anatomic location have relatively thin walls without smooth muscle fibers and valves ${ }^{16,17}$, which makes them vulnerable for rupture. If the sinus into which they drain is occluded, dilatation and reversal of the direction of blood flow will likely occur, leading to reflux overflow hemorrhage in the thalamus and in the subarachnoid space around the deep cerebral venous system. Deep cerebral venous thrombosis may produce extensive venous congestion and vasogenic edema, ultimately leading to venous infarction as well as PSH.

The second patient had fulfilled the criteria for Behçet's disease according to the International Study Group diagnostic guidelines $^{18}$. The majority of patients with neuro-Behçet's disease have a clinical picture of aseptic meningoencephalitis with brainstem involvement ${ }^{19}$. The most prevalent abnormalities are located in the mesodiencephalic junction, followed by basal ganglia and the thalamus, according to the MRI findings for neuro-Behçet's disease ${ }^{20,21}$. Regional hemodynamic properties characterized by absence of collateral venous structures may help explain this preferential geographic involvement ${ }^{22}$. Autopsy studies and biopsy specimens of the CNS lesions display vasculitis as the underlying pathology with clear evidence for venous predominance that often accompanies venous thrombosis ${ }^{21,22}$. This evidence supports the notion that the pathogenesis of neuro-Behçet's disease is primarily an underlying venulitis. The association of PSH and neuro-Behçet's in this case may be less clear since the digital subtraction angiography did not reveal any evidence of a vasculitis or venous abnormality. However, the clinical suspicion for the recurrence of neuro-Behçet's disease was very high based on the CSF findings, fever and elevated inflammatory markers without any evidence of infection. Underlying venulitis from neuro-Behçet's disease in this patient may have been an important predisposing factor for the development of PSH.

Although the third patient did not fulfill the diagnostic criteria for Behçet's disease, recurrent oral ulcerations occurring at least three times a year, and papulopustular skin lesions on the venupuncture sites strongly suggest a possibility of Behçet disease, incomplete type, according to the Japanese criteria revised in $1987^{23}$. It is unclear if the episodic ocular inflammation were self-limited uveitis or just a simple viral conjunctivitis. Similar to the second patient, underlying central nervous system (CNS) vasculitis with venous predominance may have predisposed the patient for the thrombosis in the straight sinus and the vein of Galen, causing extensive venous congestion and vasogenic edema, leading to venous infarction as well as PSH.

All of our illustrated cases of PSH can be readily explained by the underlying abnormalities in the venous circulation. PSH in association with cavernous sinus thrombosis ${ }^{24}$ and jugular venous occlusion ${ }^{25}$ have also been recently reported. Underlying venous pathology appears to be a common denominator and may have been an important predisposing factor for the development of PSH. These illustrated cases represent a distinct subtype of PSH and are clearly different from the commonly encountered $\mathrm{PSH}$ with an excellent outcome and without any evidence of underlying venous pathology. However, despite the limitations, the pathophysiologic mechanism leading to PSH in our cases could provide theoretical clues to the anatomic origin of PSH in general. The underlying venous pathology from recent reports of PSH $^{15,24,25}$ combined with our case series provides better understanding of this clinical entity of unknown etiology and provides insight into the anatomic origin of PSH.

\section{REFERENCES}

1. Marquardt G, Niebauer T, Schick U, Lorenz R. Long term follow up after perimesencephalic subarachnoid haemorrhage. J Neurol Neurosurg Psychiatry. 2000;69(1):127-30.

2. Bradac GB, Bergui M, Ferrio MF, Fontanella M, Stura G. Falsenegative angiograms in subarachnoid haemorrhage due to intracranial aneurysms. Neuroradiology. 1997;39(11):772-6.

3. Schwartz TH, Solomon RA. Perimesencephalic nonaneurysmal subarachnoid hemorrhage: review of the literature. Neurosurgery. 1996;39(3):433-40; discussion 40.

4. Rinkel GJ, Wijdicks EF, Hasan D, Kienstra GE, Franke CL, Hageman LM, et al. Outcome in patients with subarachnoid haemorrhage and negative angiography according to pattern of haemorrhage on computed tomography. Lancet. 1991;338 (8773):964-8.

5. van Gijn J, van Dongen KJ, Vermeulen M, Hijdra A. Perimesencephalic hemorrhage: a nonaneurysmal and benign form of subarachnoid hemorrhage. Neurology. 1985;35(4): 493-7.

6. Rinkel GJ, Wijdicks EF, Vermeulen M, Hasan D, Brouwers PJ, van Gijn J. The clinical course of perimesencephalic nonaneurysmal subarachnoid hemorrhage. Ann Neurol. 1991;29(5):463-8.

7. Schwartz TH, Mayer SA. Quadrigeminal variant of perimesencephalic nonaneurysmal subarachnoid hemorrhage. Neurosurgery. 2000;46(3):584-8.

8. Rinkel GJ, Wijdicks EF, Vermeulen M, Ramos LM, Tanghe HL, Hasan D, et al. Nonaneurysmal perimesencephalic subarachnoid hemorrhage: CT and MR patterns that differ from aneurysmal rupture. AJNR Am J Neuroradiol. 1991;12(5):829-34. 
9. van der Schaaf IC, Velthuis BK, Gouw A, Rinkel GJ. Venous drainage in perimesencephalic hemorrhage. Stroke. 2004;35 (7):1614-8

10. Watanabe A, Hirano K, Kamada M, Imamura K, Ishii N, Sekihara $\mathrm{Y}$, et al. Perimesencephalic nonaneurysmal subarachnoid haemorrhage and variations in the veins. Neuroradiology. 2002; 44(4):319-25.

11. Yamakawa $\mathrm{H}$, Ohe $\mathrm{N}$, Yano $\mathrm{H}$, Yoshimura S, Iwama T. Venous drainage patterns in perimesencephalic nonaneurysmal subarachnoid hemorrhage. Clin Neurol Neurosurg. 2008;110 (6):587-91

12. Daenekindt T, Wilms G, Thijs V, Demaerel P, Van Calenbergh F. Variants of the basal vein of Rosenthal and perimesencephalic nonaneurysmal hemorrhage. Surg Neurol. 2008;69(5):526-9; discussion 9.

13. Matsumaru Y, Yanaka K, Muroi A, Sato H, Kamezaki T, Nose T. Significance of a small bulge on the basilar artery in patients with perimesencephalic nonaneurysmal subarachnoid hemorrhage. Report of two cases. J Neurosurg. 2003;98(2): 426-9.

14. Lansberg MG. Concurrent presentation of perimesencephalic subarachnoid hemorrhage and ischemic stroke. J Stroke Cerebrovasc Dis. 2008;17(4):248-50.

15. Sztajzel R, Coeytaux A, Dehdashti AR, Delavelle J, Sinnreich M. Subarachnoid hemorrhage: a rare presentation of cerebral venous thrombosis. Headache. 2001;41(9):889-92.

16. Capra NF, Anderson KV. Anatomy of the cerebral venous system. In: Kapp JP, Schmidek HH, editors. The cerebral venous system and its disorders. Orlando, FL: Grune \& Stratton; 1984. p. 1-36.

17. Ameri A, Bousser MG. Cerebral venous thrombosis. Neurol Clin. 1992;10(1):87-111.
18. International Study Group for Behcet's Disease. Criteria for diagnosis of Behçet's disease. Lancet. 1990;335(8697):1078-80.

19. Akman-Demir G, Serdaroglu P, Tasci B. Clinical patterns of neurological involvement in Behcet's disease: evaluation of 200 patients. The Neuro-Behçet's Study Group. Brain. 1999;122 (Pt 11):2171-82

20. Banna M, el-Ramahl K. Neurologic involvement in Behcet disease: imaging findings in 16 patients. AJNR Am J Neuroradiol. 1991;12(4):791-6.

21. Kocer N, Islak C, Siva A, Saip S, Akman C, Kantarci O, et al. CNS involvement in neuro-Behçet's syndrome: an MR study. AJNR Am J Neuroradiol. 1999;20(6): 1015-24.

22. Kidd D, Steuer A, Denman AM, Rudge P. Neurological complications in Behçet's syndrome. Brain. 1999;122 (Pt 11): 2183-94.

23. Mizushima Y. Recent research into Behçet's disease in Japan. Int J Tissue React. 1988;10(2):59-65.

24. Kurosu A, Suzukawa K, Amo M, Horinaka N, Arai H. Perimesencephalic non-aneurysmal subarachnoid hemorrhage caused by cavernous sinus thrombosis: case report. Neurol Med Chir (Tokyo). 2007;47(6):258-60.

25. Sangra MS, Teasdale E, Siddiqui MA, Lindsay KW. Perimesencephalic nonaneurysmal subarachnoid hemorrhage caused by jugular venous occlusion: case report. Neurosurgery. 2008;63(6):E1202-3; discussion E3. 